\title{
Thermotropic Liquid Crystals of Main-Chain Polyesters with a Mesogenic 4,4'-Biphenyldicarboxylate Unit XII. Unusual Molecular Orientation in Fibers Drawn from Smectic Melt
}

\author{
Masatoshi Tokita, Kensuke Osada, Susumu Kawauchi, \\ and Junji WATANABE \\ Department of Polymer Chemistry, Tokyo Institute of Technology, \\ Ookayama, Meguro-ku, Tokyo 152-8552, Japan
}

(Received February 24, 1998)

\begin{abstract}
KEY WORDS Main-Chain Liquid Crystal Polyester / Smectic Phase / X-Ray Pattern / Flow Orientation / Stress-Strain Curve / Fiber /
\end{abstract}

In this series of studies, ${ }^{1-10}$ we have treated the following main-chain liquid crystal polyesters designated as $\mathrm{BB}-n$

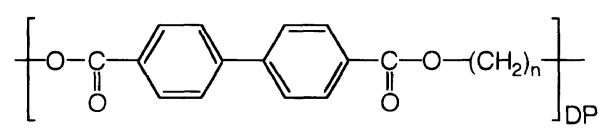

where $n$ is the carbon number of the methylene spacer. These BB- $n$ polyesters form smectic mesophases when $n$ varies from 3 to 9 . In BB- $n$ with an even $n$, a smectic $\mathrm{A}$ $\left(S_{A}\right)$ phase is formed with both axes of the polymer chain and biphenyl mesogen lying perpendicular to the layers. In contrast, BB- $n$ with an odd $n$ form a smectic $C A\left(\mathrm{~S}_{\mathrm{CA}}\right)$, in which the tilt direction of the mesogenic group is the same in all second layers but opposite between neighboring layers. ${ }^{1-3,8,9}$ A smectic $\mathrm{C}\left(\mathrm{S}_{\mathrm{C}}\right)$ phase can be prepared by the introduction of one branched methyl group into the spacer part of the even numbered BB- $n .{ }^{4}$ Typical example is BB-4(1-Me)

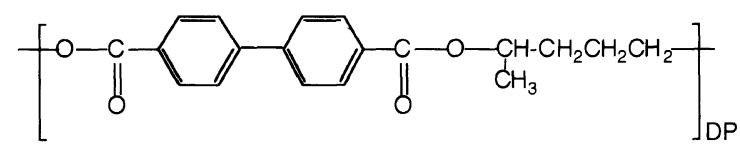

In this $S_{C}$ phase, both axes of polymer chain and mesogen are tilted to the layer normal. ${ }^{5}$ Chiral $\mathrm{S}_{\mathrm{CA}}{ }^{*}$ and $\mathrm{S}_{\mathrm{C}}$ * have also been prepared in the same homologue by giving the chiral spacer. ${ }^{2,6}$

On exploring the smectic structural properties characteristic to the main-chain type of polymers using these smectic materials, we have found that $S_{A}$ phases show anomalous molecular orientation when flow deformation is applied. ${ }^{6-10,11}$ The polymer chains align perpendicular to the flow direction! ${ }^{6,11}$ Similar orientation of smectic phase has been reported by Alt, et al. ${ }^{12}$ and Leland, et $a l .{ }^{13}$ Such orientation is in contrast to that in the nematic phase, in which the polymer chains lie parallel to the flow direction, and is surely characteristic of polymeric smectic phases. This paper extends the study of flow behavior of other smectic phases, $S_{C A}$ and $S_{C}$ phases. Such an unusual orientation was noted commonly in $\mathrm{S}_{\mathrm{A}}$, $\mathrm{S}_{\mathrm{CA}}, \mathrm{S}_{\mathrm{C}}$ phases.

\section{EXPERIMENTAL}

Three representative polyesters, BB-6, BB-5, and BB-4(1-Me), which can form the $S_{A}, S_{C A}$, and $S_{C}$, respectively, were used. These polyesters were synthesized by melt transesterification from dimethyl $p, p^{\prime}$-bibenzoate and corresponding diols with isopropyl titanate as catalyst. The inherent viscosities of BB-6, BB-5, and BB-4(1-Me) are $0.49,0.52$, and $0.46 \mathrm{dL} \mathrm{g}^{-1}$, respectively, as measured at $30^{\circ} \mathrm{C}$ by using $0.5 \mathrm{~g} \mathrm{dL}^{-1}$ solutions in a $60 / 40 \mathrm{w} / \mathrm{w}$ mixture of phenol and tetrachloroethane.

X-Ray diffraction patterns were observed using a Rigaku-Denki RU-200 BH with Ni-filtered $\mathrm{Cu}-K_{\alpha}$ radiation. Stress-strain curves were obtained on a Seiko Instruments TMA SSC5200 with a strain rate of $50 \mu \mathrm{m}$ $\min ^{-1}$.

\section{RESULTS AND DISCUSSION}

Figures 1(a), 1(b), and 1(c) show X-ray patterns for BB-6, BB-5, and BB-4(1-Me) fibers, respectively, taken at a room temperature. The fiber specimens were prepared by drawing the isotropic melt at a rate of about $20 \mathrm{~cm} \mathrm{~s}^{-1}$. In BB-6, the crystallization takes place even in as-span fibers so that the X-ray pattern of Figure 1(a) includes the sharp inner reflections on meridian attributed to the layered structure and several other reflections associated with crystal. In BB-5 and BB-4(1-Me) fibers, X-ray patterns characteristic of the mesophases are observed even at a room temperature because of very late crystallization. ${ }^{10}$ In Figure 1(b) for BB-5 fiber, the sharp inner reflection is found on meridian while the broad reflections are split above and below the equator, leading to the $\mathrm{S}_{\mathrm{CA}}$ structure. ${ }^{2}$ In Figure 1(c) for BB-4(1$\mathrm{Me}$ ), inner reflections are split into two positions around meridian and broad reflections are centered on equator. These characteristic diffraction patterns are attributable to the $\mathrm{S}_{\mathrm{C}}$ structure. ${ }^{4,5}$ The tilt angle of both axes of mesogens and polymer chain to the layer normal is estimated as $30^{\circ}$ from the splitting angle of the layer reflection. The corresponding layer structures in the fibers are illustrated in Figures 1(d)-1(f). In all these fibers, the polymer chain axis lies parallel to the fiber direction.

The fibers can be drawn from smectic melts if flow elongation is applied at a relatively low rate of $1-2$ 
(a)

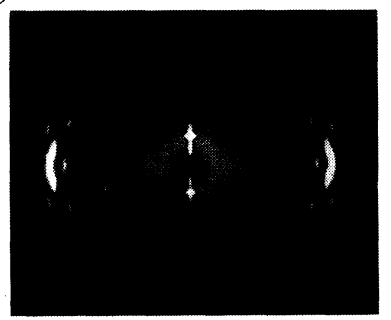

(b)

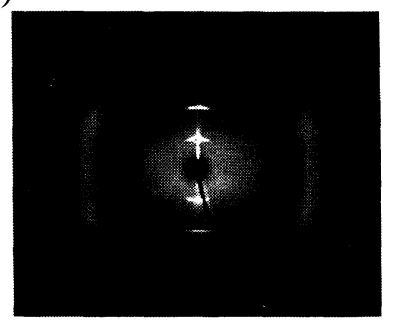

(c)

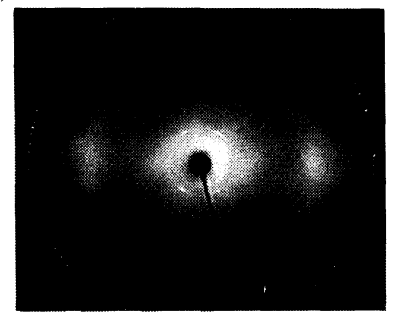

(d)

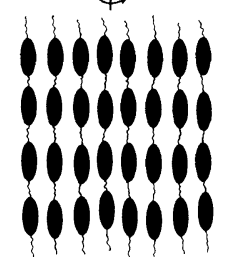

(e)

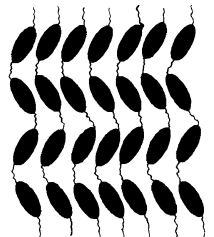

(f)

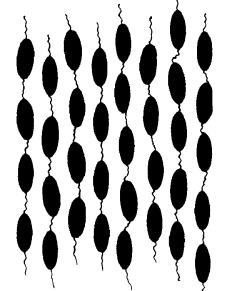

Figure 1. X-Ray photographs for (a) BB-6, (b) BB-5, and (c) $\mathrm{BB}-4(1-\mathrm{Me})$ fibers drawn from the isotropic melt at a rate of about $20 \mathrm{~cm} \mathrm{~s}^{-1}$. The fiber axis is in the vertical direction. The molecular arrangements in the fibers deduced from the X-ray patterns are illustrated in parts (d), (e), and (f).

$\mathrm{cm} \mathrm{s}^{-1}$. X-ray photographs are shown in Figures 2(a)2(c). In comparison with the patterns of Figures 1(a)1(c), one can find the several distinct features. The patterns of the BB-6 and BB-5 fibers shown in Figures 2(a) and 2(b) are similar to the patterns of Figures 1(a) and $1(b)$, respectively, but quite different in the orientation geometry of reflections with respect to the fiber axis. The layer reflections in smectic melt-span fibers appear on the equator, showing that the smectic layers lie parallel to the fiber axis, i.e., the polymer chain axis orients perpendicular to the elongation flow direction. X-ray pattern of Figure 2(c) for the BB-4(1-Me) fiber looks completely different from that in Figure 1(c). The smectic layer reflections are centered on the equator and the broad reflections are split on both sides of meridian. These can be accounted for by the $S_{C}$ structure with the layers lying parallel to the fiber axis. From the splitting angles of the outer broad reflections, the tilt angle of the $\mathrm{S}_{\mathrm{C}}$ can be estimated as around $30^{\circ}$ which coincides with that estimated from the splitting angle of the layer reflections in Figure 1(c). ${ }^{9}$ Corresponding smectic structures are illustrated in Figures 2(d)-2(f). From these figures, we can safely conclude that elongation flow of a smectic melt arranges the smectic layers parallel to the flow direction.

The fibers drawn from the smectic melts can be further elongated even at room temperature. Figure 3 shows the 688

(a)

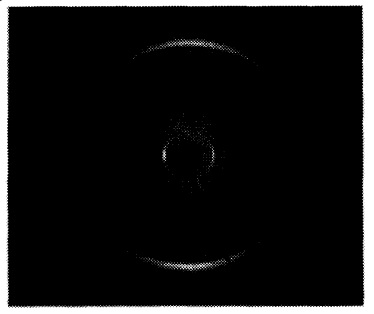

(d)

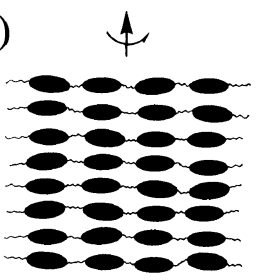

(b)

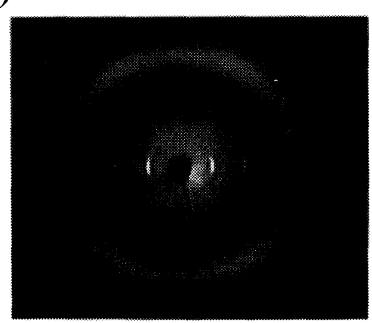

(e)

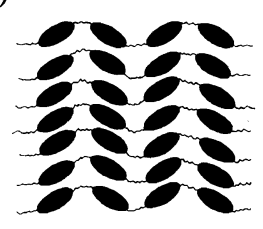

(f)
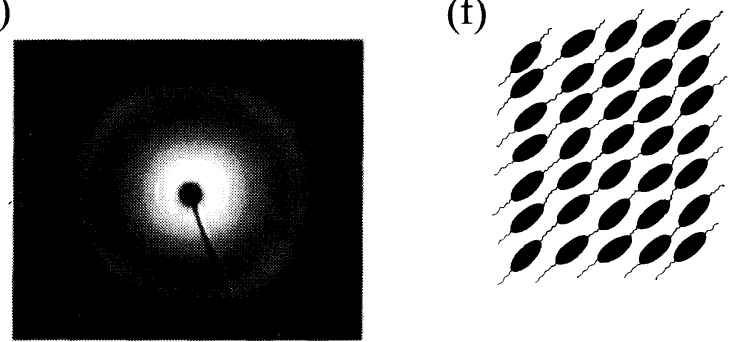

Figure 2. X-Ray photographs for (a) BB-6, (b) BB-5, and (c) $\mathrm{BB}-4(1-\mathrm{Me})$ fibers drawn from the smectic melt at a rate of about $1 \mathrm{~cm} \mathrm{~s}^{-1}$. The fiber axis is in the vertical direction. The molecular arrangements in the fibers deduced from the X-ray patterns are illustrated in parts (d), (e), and (f).

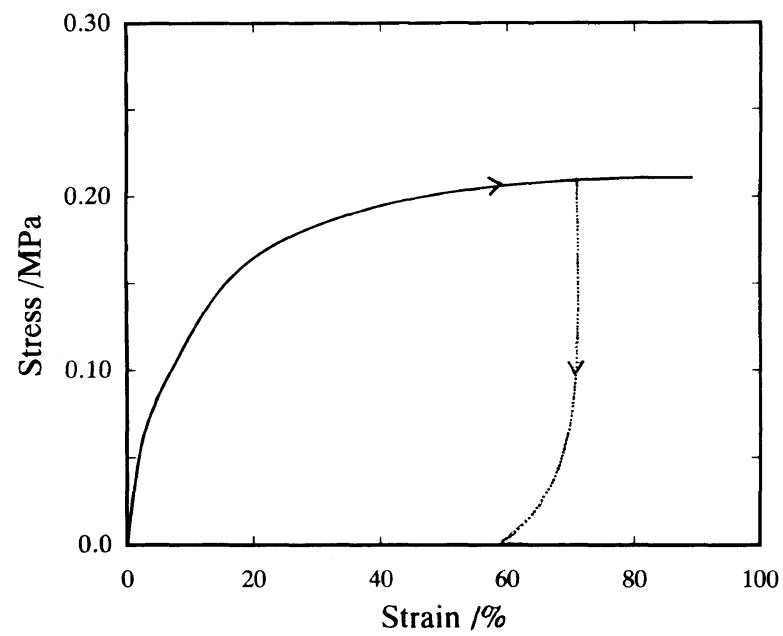

Figure 3. Stress-strain curves of a BB-5 fiber drawn from the $S_{C A}$ melt. Solid curve was observed up to the breaking point and the dotted one is the relaxation curve observed on unloading at the strain of $70 \%$.

stress-strain curve measured for the BB-5 fiber with a diameter of $0.2 \mathrm{~mm}$. This fiber is composed only of the $\mathrm{S}_{\mathrm{CA}}$ glass as mentioned above. The glass transition temperature is around $45^{\circ} \mathrm{C}$ higher than room temperature. ${ }^{10}$ Stress is proportional to the strain at an initial stage of strain until $\varepsilon=2.5 \%$. Apparent modulus determined with the slope is around $0.25 \mathrm{GPa}$. The fiber 
(a)

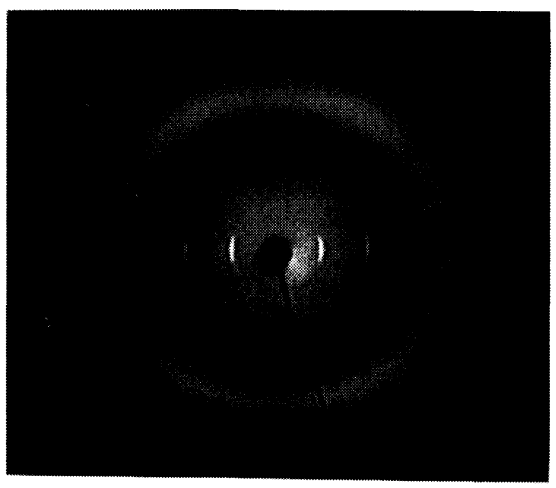

(b)

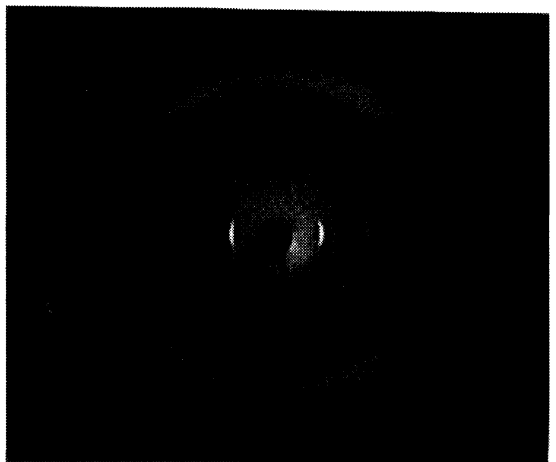

Figure 4. Wide-angle X-ray diffraction patterns of BB-5 fiber drawn from the $\mathrm{S}_{\mathrm{CA}}$ melt taken under the strains of (a) $0 \%$ and (b) $70 \%$. The fiber axis is in the vertical direction.

is stretched up to $\varepsilon=100 \%$ with constant stress of 0.2 MPa and breaks. On unloading at $\varepsilon=70 \%$ before the fiber breaks, almost all the strain remains as given by the dotted curve in Figure 3, showing that the strain applied results from the permanent deformation. The X-ray pattern taken at a strain of $70 \%$ is shown in Figure 4(b). The pattern is not essentially altered from that of unloading fiber shown in Figure 4(a), although only a few percent reduction is observed in the layer spacing. This indicates that elongation includes neither deformation in the smectic layer nor change in the molecular orientation. Thus, elongation takes place as if the smectic layers slip to each other so as to lie parallel to the fiber axis.

Actually, such flow has been observed in low molecular weight smectic phases where each smectic layer is composed of the molecules ${ }^{14}$ (refer to Figure 5(a)). In the polymeric system, this mechanism is not acceptable since the mesogenic groups in neighboring layers are linked to each other through a flexible spacer and so each polymer molecule passes through many smectic layers. To explain this orientation, we postulate that lamellar domains are formed in the smectic field and flow takes place along domain boundaries ${ }^{6}$ (see Figure 5(b)).

The stacked lamellar structure was found in crystals formed from the $\mathrm{S}_{\mathrm{A}}$ phase by the SAXS method. ${ }^{6,7,15}$ The lamellar spacings are centered around $250 \AA$, corresponding to 15 times the length of the repeat unit so that an appreciable number of chain foldings are included in a chain. Since we can hardly consider that chain folding takes place on a crystallization from the $\mathrm{S}_{\mathrm{A}}$ phase, we assume that it already exists in the smectic field. This is reasonable, considering the entropy force (a)

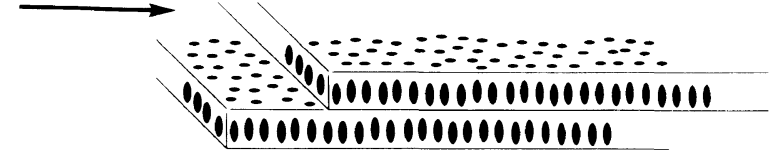

(b)

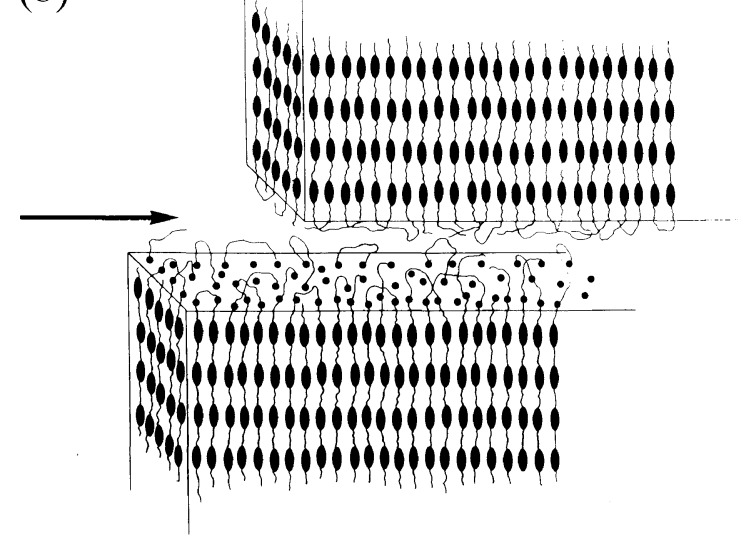

Figure 5. Schematic comparison of the flow of (a) the low molecular mass smectic phase and (b) polymeric smectic phase. In the polymeric system, chain folded lamellae are formed and slip over each other while in the low molar mass system, smectic layers can slip over each other.

as following. ${ }^{16}$ To produce the orientational and positional orders in smectic phases, the obvious configuration for a polymer molecule to assume in order to minimize its energy is to lie entirely along the director. On the other hand, the energy penalty due to the chain folding should be smaller in this type of main-chain polymers with the flexible spacer. Thus, the chain folding can be considered to arise as a result of the counterbalance between its energetical cost and entropy gain where it can be placed along the chain length. This consideration leads to the suggestion that the correlation length of chain folding decreases with increasing temperature. $^{16}$ In fact, we have observed that lamellar spacing of crystal becomes to be larger when crystallization takes place at the lower temperature of the $S_{A}$ phase. ${ }^{7}$ This dependence of the crystal lamellar size on the crystallization temperature is obviously opposite to that observed for conventional polymers.

Thus, the mechanism given in Figure 5(b), i.e., the mutual slip of the lamellae, is valid for the anomalous flow behavior. Although the reflection maxima in SAXS to clarify the existence of lamellar domains have never been observed for the smectic phase or the quenched smectic glass, ${ }^{6,15}$ this may result from the poor contrast of electron density between the inside and outside regions of lamellar block.

\section{CONCLUSIONS}

The unusual orientations of molecules were noted in 
the fibers drawn from the $\mathrm{S}_{\mathrm{A}}, \mathrm{S}_{\mathrm{CA}}$, and $\mathrm{S}_{\mathrm{C}}$ melts of $\mathrm{BB}$-polyesters. In all cases, smectic layers orient parallel to the fiber axis and the polymer chains lie perpendicular or tilted to the fiber axis. This anomalous flow orientation can be explained if chain folded lamellae are formed in the smectic phases and slip over each other.

\section{REFERENCES}

1. J. Watanabe and M. Hayashi, Macromolecules, 21, 278 (1988); ibid., 22, 4083 (1989).

2. J. Watanabe and S. Kinoshita, J. Phys. II (France), 2, 1237 (1992).

3. J. Watanabe, M. Hayashi, A. Morita, and T. Niori, Mol. Cryst. Liq. Cryst., 254, 221 (1994)

4. J. Watanabe, M. Hayashi, S. Kinoshita, and T. Niori, Polym. J., 24, 597 (1992)

5. J. Watanabe, M. Hayashi, and M. Tokita, Reactive \& Functional Polymers, 30, 191 (1996).
6. M. Tokita, T. Takahashi, M. Hayashi, K. Inomata, and J Watanabe, Macromolecules, 29, 1345 (1996).

7. M. Tokita, K. Osada, and J. Watanabe, Liq. Cryst., 23, 453 (1997).

8. J. Watanabe, M. Hayashi, T. Niori, M. Tokita, and Y. Nakata, Prog. Polym. Sci., 22, 1053 (1997).

9. M. Tokita, K. Osada, and J. Watanabe, Liq. Cryst., 24, 477 (1998).

10. M. Tokita, K. Osada, and J. Watanabe, Polym. J., 30, 589 (1998)

11. W. R. Krigbaum and J. Watanabe, Polymer, 24, 1229 (1983).

12. D. J. Alt, S. D. Hudson, R. O. Garay, and K. Fujishiro, Macromolecules, 28, 1575 (1995).

13. M. Leland, Z. Wu, M. Chhajer. R-M. Ho, S. Z. D. Cheng, A. Keller, and H. R. Kricheldorf, Macromolecules, 30, 5249 (1997).

14. G. W. Gray and J. W. Goodby, "Smectic Liquid Crystals," Leonard Hill, Glasgow, 1984.

15. T. Takahashi and F. Nagata, J. Macromol. Sci. B, Phys., B28, 34 (1989)

16. D. R. M. Williams and M. Warner, J. Phys. (France), 51, 317 (1990). 